\title{
In this issue
}

By John Ashkenas, Science Editor

\author{
A regulatory subunit of protein kinase $A$ is the tumor suppressor lost in Carney complex \\ (See article on pages R31-R38)
}

Carney complex, a heritable cell-overgrowth syndrome that goes by several aliases, presents with benign pigmented growths in the skin and causes endocrine dysfunction and, most seriously, obstructive myxomas in the heart. Linkage analyses over the last 5 years have found evidence for at least two genes that can cause this syndrome. Now, Casey et al. have identified one of these genes, $P R K A R 1 \alpha$, which encodes a regulatory subunit of protein kinase $\mathrm{A}$, a ubiquitous intracellular signaling protein. Having determined that a subset of families with this condition shows linkage to 17q24, Casey and coworkers screened affected individuals for coding mutations in PRKAR1 $\alpha$, which maps to this locus. They report three distinct frameshift mutations, all of which are apparent null alleles. Although haploinsufficiency for this gene is clearly involved in Carney pathogenesis, the subsequent events are unclear, since the authors found no evidence that the remaining PRKAR1 $\alpha$ allele is mutated or silenced in a cardiac myxoma from one of their probands. Casey et al. speculate that some second hit at an unlinked locus drove cell proliferation in this tissue. The identity of the other putative Carney complex gene and its relationship to PRKAR1 $\alpha$ remain to be discovered.

\section{Defining a cell death pathway in the ischemic brain} (See article on pages 643-653)

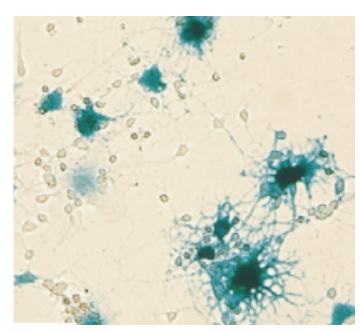

The central nervous system (CNS) is well known to be sensitive to the effects of oxygen deprivation, such as occurs during strokes. Still, the mechanisms by which the various CNS cell types die during ischemic episodes are far from clear. Ischemic conditions kill cells in the brain both directly, as a result of hypoxia and ATP depletion, and indirectly, through the release of toxic levels of neurotransmitters. In addition, both apoptotic and necrotic death pathways have been proposed. Shibata et al. have developed transgenic mouse lines in which to study the effects of cerebral ischemia on oligodendrocytes (OLGs), the myelinating cells of the CNS, which are particularly sensitive to damage during strokes. By driving expression of the broad-spectrum caspase inhibitor p35 specifically to OLGs, Shibata and colleagues prevent death of these cells under ischemic conditions, both in vitro and in vivo. In particular, the authors implicate caspase-11, acting upstream of caspase-3, in the death of these cells, and they show that OLGs in the brains of caspase- $11^{-/-}$mice survive long periods of focal ischemia. For more on ischemic injury in the brain and elsewhere, see the Perspective series beginning in this issue of the JCI.
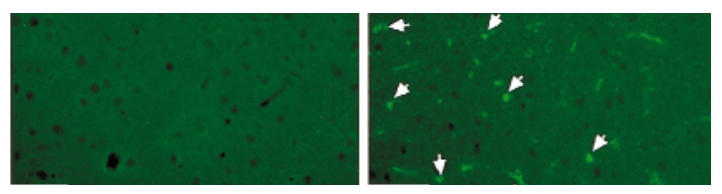

\section{Transcriptional regulation in sunburned skin}

(See article on pages 663-670)

The extracellular matrix in the skin is a surprisingly dynamic environment, easily altered by changes in the rate of biosynthesis or turnover of collagens and other component proteins. Fisher and colleagues previously showed that ultraviolet (UV) irradiation causes metalloproteinase activation and thus promotes the degradation of dermal collagens. Here, they show that even relatively low doses of UV irradiation, sufficient to cause some reddening of the skin, also cause a decrease in the rate of collagen synthesis in treated areas of the skin relative to adja-

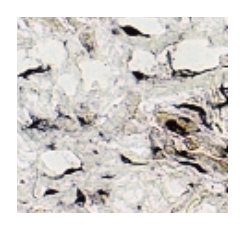
cent untreated regions. This effect is seen within 8 hours of treatment and occurs at the level of transcription; processing of newly synthesized collagens appears to be unaffected. Cultured skin fibroblasts show a similar effect of UV treatment, allowing Fisher et al. to study regulation of this response by the transcription factor AP-1, which is known to bind the promoter region of procollagen gene COL1A2. The authors confirm that this transcription factor is required for UV-dependent silencing of the COL1A2 promoter, and they further show that pretreatment of the skin with retinoic acid, which prevents activation of AP-1, blocks the pathway and allows for continued synthesis of type I collagen after UV treatment. The UV-induced silencing of collagen gene expression and induction of MMP expression appear to be under control of identical or overlapping signaling pathways. Retinoic acid apparently blunts both effects, suggesting that this agent, which has been marketed for its ability to block photoaging of the skin, probably acts by both mechanisms to help maintain the quantity and structure of collagens in the skin. 